\title{
Singularly perturbed elliptic problems of second order with a singular line
}

\author{
H.-G. Roos and L. Tobiska
}

Für einige Klassen singulär gestörter elliptischer Probleme, wo die Charakteristiken des reduzierten P'roblems parallel oder senkrecht zur singulären Linie verlaufen, werden gleichmäßige asymptotische Approximationen konstruiert. Die Approximationsordnung ist abhängig vom Verhalten der Koeffizienten des reduzierten Problems in Umgebung der singulären Iinie und von den Eigenschaften des betrachteten Gebietes.

Для некоторых классов сингулярно-возмущённых әллиптических задач, в которых характеристики вырожденио й задачи параллельны или перпендикулярны $к$ особой кривой,, строятся равномерно-асимптотические аппроксимации. Порядок аппроксимации зависит от свойств коэффициентов вырожденной задачи в окрестности особой кривой и от характера рассматриваемой области.

For some classes of singularly perturbed elliptic problems, in which the characteristics of the reduced problem are parallel or perpendicular to the singular line, uniform asymptotic approximations are constructed. The order of approximation depends on the behaviour of the coefficients of the reduced problem in the neighbourhood of the singular line and on the. properties of the considered domain.

\section{Introduction}

We consider boundary value problems of the form'

$$
\begin{aligned}
& L_{\varepsilon} u_{\varepsilon}=\varepsilon L_{1} u_{\varepsilon}+L_{0} u_{\varepsilon}=0 \text { in } \Omega \\
& u_{\varepsilon}=g \quad ; \quad \text { in } \partial \Omega,
\end{aligned}
$$

where $L_{1}$ denotes a linear uniformly elliptic second order differential operator:

$$
L_{1} \equiv \sum_{i, j=1}^{2} a_{i j} \frac{\partial^{2}}{\partial x_{i} \partial x_{j}}+\sum_{i=1}^{2} a_{i} \frac{\partial}{\partial x_{i}}+a_{0} \quad\left(a_{0} \geqq 0\right),
$$

$L_{0}$ is a linear first order differential operator :

$$
L_{0} \equiv \sum_{i=1}^{2} b_{i} \frac{\partial}{\partial x_{i}}
$$

and $\varepsilon$ is a small positive parameter.

$\Phi_{\varepsilon}$ is called uniform asymptotic approximation of $u_{\varepsilon}$ on the subdomain $\tilde{\Omega} \subset \bar{\Omega}$ if the inequality

$$
\left\|u_{\varepsilon}-\Phi_{\varepsilon}\right\|_{\bar{\Omega}}=\sup _{x \in \bar{\Omega}}\left|u_{\varepsilon}(x)-\Phi_{\varepsilon}(x)\right| \leqq k_{\varepsilon}{ }^{\sigma} \quad(\sigma>0)
$$

is satisfied for a positive constant $K$ independent of $\varepsilon$. In case of $\widetilde{\Omega}=\bar{\Omega}$. we shall omit the subskript $\widetilde{\Omega}$ and only write $\|\cdot\|$. The asymptotic behaviour of the solution $u_{s}$ 
of (1.1) depends on the characteristics of $L_{0}$, in particular on the existence of singular (or turning) points. A point $\dot{x}^{*} \in \bar{\Omega}$ is called a singular point if and only if $b_{i}\left(x^{*}\right)=0$ for $i=1,2$. For isolated singular points the asymptotic behaviour of the solution $u_{\varepsilon}$ of $(1.1)$ have been investigated by many authors in detail (see, for instance $[2,4,8,10-13])$. In the case of a singular line, up to now, only very special problems of the type described above have been considered $[1,3,5-7,16]$.

Our main objective is to construct uniform approximations of the solution of typical classes of (1.1) in which the characteristics of $L_{0}$ are parallel or perpendicular to the singular line. For this purpose let us assume that $x_{2}=0$ is the singular line and that $\Omega$ can be described by

$$
\Omega^{\prime}=\left\{\left(x_{1}, x_{2}\right) \mid-1<x_{1}<+1,-f_{1}\left(x_{1}\right)<x_{2}<f_{2}\left(x_{1}\right)\right\}
$$

or

$$
\Omega=\left\{\left(x_{1}, x_{2}\right) \mid-1<x_{1}<+1,0<x_{2}<f_{2}\left(x_{1}\right)\right\}
$$

by means of smooth functions $f_{1}, f_{2}$ with $f_{1,2}( \pm 1)=0$ and $f_{1,2}\left(x_{1}\right)>0$ on the open interval $(-1,+1)$. As a result $\Omega$ is an admissible domain in the sense of $[15]$ and the existence of a uniquely determined classical solution $u_{\varepsilon}$ of (1.1) can be guaranteed for sufficiently. smooth coefficients of $L_{1}$ 'and $L_{0}$.

\section{A singular line perpendicular to the characteristies}

We consider the singularly perturbed problem

$$
\begin{aligned}
& L_{\varepsilon} u_{\varepsilon} \equiv \dot{\varepsilon} L_{1} u_{\varepsilon} \pm x_{2}{ }^{l} \frac{\partial u_{\varepsilon}}{\partial x_{2}}=0 \text { in } \Omega \\
& u_{\varepsilon}=g \text { on } \partial \ddot{\Omega} \text {. }
\end{aligned}
$$

A uniform asymptotic approximation will be constructed by applying the method of matched asymptotic expansion. In dependence on the behaviour of the characteristics near the boundary we use following notations for the smooth parts of the boundary:

$$
I_{ \pm}=\{x \in \partial \Omega \mid b(x) \cdot v(x) \gtreqless 0\}, \cdot \Gamma_{0}=\{x \in \partial \Omega \mid b(x) \cdot v(x)=0\} ;,
$$

where $b=\left(b_{1}, b_{\dot{2}}\right)$ and $v$ denotes the outward directed unit normal.

First we assume that

$$
\Omega=\left\{\left(x_{1}, x_{2}\right) \mid-1<x_{1}<+1,-f_{1}\left(x_{1}\right)<x_{2}<f_{2}\left(x_{1}\right)\right\}
$$

Now, let $f_{1,2}$ vanish at $x_{1}= \pm 1$ of the order $m_{1,2}^{ \pm}$,

$$
\left.g\right|_{x_{2}=f_{2}\left(x_{1}\right)}=g_{2}\left(x_{1}\right),\left.\quad g\right|_{x_{2}=-f_{1}\left(x_{1}\right)}=g_{1}\left(x_{1}\right)
$$

and $l$ be even. Then, without restriction of generality, we can assume that $L_{0}=-x_{2}{ }^{2 k} \frac{\partial}{\partial x_{2}}$ and it holds

$$
\Gamma_{-}=\left\{\left(x_{1}, x_{2}\right) \mid \dot{x_{2}}=f_{2}\left(x_{1}\right)\right\}, \quad \Gamma_{+}=\left\{\left(x_{1}, x_{2}\right) \mid x_{2}=-f_{1}\left(x_{1}\right)\right\} .
$$

Consequently, the solution of the global problem becomes $g_{2}\left(x_{1}\right)$, such that the boundary condition on $\Gamma_{+}$does not satisfied and local correctors are needed. 
In the neighbourhood of $\Gamma_{+}$let $\zeta=\frac{x_{2}+f_{1}\left(x_{1}\right)}{\varepsilon}$ and

$$
L_{\varepsilon}=\varepsilon^{-1}\left(-a\left(x_{1}\right) \frac{\partial^{2}}{\partial \zeta^{2}}-f_{1}^{\prime}\left(x_{1}\right) \frac{\partial}{\partial \zeta}\right)+\check{L_{\varepsilon}^{*}} .
$$

Constructing a local corrector $v=v_{0}+\varepsilon v_{1}$, with $\left|L_{\varepsilon} v\right|^{\prime} \leqq K \cdot \varepsilon$ such that the boundary conditions on $\Gamma_{+}$are satisfied by $v+g_{2}$ we obtain

$$
v_{0}\left(x_{1}, \zeta\right)=\left[g_{1}\left(x_{1}\right)-g_{2}\left(x_{1}\right)\right] \exp \left(-\frac{f_{1}^{\prime}\left(x_{1}\right)}{a\left(\dot{x}_{1}\right)} \cdot \frac{f_{1}\left(x_{1}\right)+x_{2}}{\varepsilon}\right) .
$$

On $\Gamma_{-} v_{0}$ satisfies $\left.v_{0}\right|_{-}=\left(g_{1}-g_{2}\right) \exp \left(-\frac{f_{1}^{\prime}}{a} \frac{f_{1}+f_{2}}{\varepsilon}\right)$ and is exponentially small for $\left|x_{1}\right| \leqq x_{0}<1$. In the neighbourhood of $x_{1}=1 v_{0} \mid r_{-}$behaves as $\mu \exp \left(-\frac{\mu^{l m_{1}+}+m \mid n\left(m_{1}{ }^{+}, m_{2}+\right.}{\varepsilon}\right)$. For arbitrary positive $r$ it holds $\left[\mu \exp \left(-\frac{\mu^{r}}{\varepsilon}\right) \mid\right.$ $\leqq K \varepsilon^{\frac{1}{r}}$, such that $\left|v_{0}\right| r_{-} \mid \leqq K \varepsilon^{\frac{1}{s_{1}}}$ with $s_{1}=\max \left(2 k m_{1}{ }^{+}+\min \left(m_{1}^{+}, m_{2}^{+}\right), 2 k m_{1}^{-}\right.$. $\left.+\min \left(m_{1}^{-}, m_{2}^{-}\right)\right)$. Summing up we obtain

$$
\left|L_{\varepsilon}\left(u_{\varepsilon}-\left(g_{2}+v\right)\right)\right| \leqq K \varepsilon \text { in } \Omega,\left|u_{\varepsilon}-\left(g_{2}+v\right)\right| \leqq K \varepsilon^{\frac{1}{s_{2}}}
$$

on $\partial \Omega$. The barrier function $K_{1} \varepsilon^{\frac{1}{\varepsilon_{1}}}-K_{2} \varepsilon^{2 k+1} v_{\iota}\left(x_{2}\right)$ with

$$
v_{c}\left(x_{2}\right)=\int_{-\infty}^{x_{2} / e^{\frac{1}{2 k+1}}} \int_{-\infty}^{q} \exp \left(\frac{p^{2 k+1}-q^{2 k+1}}{K_{3}}\right) d p d q
$$

yields the estimate, $\left|u_{\varepsilon}-\left(g_{2}+v\right)\right| \leqq K \varepsilon^{\frac{1}{s_{1}}}+K \varepsilon^{\frac{2}{2 k+1}}$ [17]. Because of $s_{1} \geqq 2 k$ +1 we obtain

The'orem 2.1: Let $L_{0} \equiv-x_{2}{ }^{2 k} \frac{\partial}{\partial x_{2}}$ and let the singular line lie in the interion of $\Omega$.

$$
\left\|u_{\varepsilon}-\left(g_{2}+v_{0}\right)\right\| \leqq K \varepsilon^{\frac{1}{\varepsilon_{1}}}
$$

with $s_{1}=\max \left(\min \left(m_{1}^{+}, m_{2}^{+}\right)+2 k m_{1}{ }^{+}, \min \left(m_{1}{ }^{-}, m_{2}{ }^{-}\right)+2 k m_{1}{ }^{-}\right)$.

Considering the subdomains $x_{2}>0$ and $x_{2}<0$, respectively, we obtain analogous problems. Let.

$$
\Omega=\left\{\left(x_{1}, x_{2}\right) \mid-1<x_{1}<+1,0<x_{2}<f_{2}\left(x_{1}\right)\right\} .
$$

Again' $\dot{g}_{2}\left(x_{1}\right)$ is 'the solution of the global problem. Setting $\zeta=\frac{x_{2}}{\frac{1}{\varepsilon^{2 k+1}}}$ it bolds

$$
L_{\varepsilon}=\varepsilon^{\frac{2 k-1}{2 k+1}}\left(-a\left(x_{1}\right) \frac{\partial^{2}}{\partial \zeta^{2}}-\zeta^{2 k} \frac{\partial}{\partial \zeta}\right)+\varepsilon^{\frac{2 k}{2 k+1}} L_{\varepsilon}^{*}
$$

Requiring

$$
a \frac{\partial^{2} v}{\partial \zeta^{2}}+\zeta^{2 k} \frac{\partial v}{\partial \zeta}=0,\left.\quad v\right|_{\zeta=0}=g_{1}\left(x_{1}\right)-g_{2}\left(x_{1}\right)
$$


we obtain

$$
v=\frac{\left(g_{1}\left(x_{1}\right)-g_{2}\left(x_{1}\right)\right)}{A} \int_{x_{2} / s^{\frac{1}{2 k^{+1}}}}^{\infty} \exp \left(-\frac{t^{2 k+1}}{a(2 k+1)}\right) d t
$$

with $A=\int_{0}^{\infty} \exp \left(-\frac{t^{2 k+1}}{a(2 k+1)}\right) d t$. Now $u_{\varepsilon}-\left(g_{2}+v\right)$ satisfies

$$
\begin{aligned}
& \left|L_{\varepsilon}\left(u_{\varepsilon}-\left(g_{2}+v\right)\right)\right| \leqq K \varepsilon^{\frac{2 k}{2 k+1}} \text { in } \Omega, \\
& u_{\varepsilon}-\left.\left(g_{2}+v\right)\right|_{x_{s}=0}=0, \\
& \left|u_{\varepsilon}-\left(g_{2}+v\right)\right|_{x_{2}=\boldsymbol{s}_{2}\left(x_{1}\right)} \mid \leqq K \varepsilon^{\frac{1}{s_{2}}}, \quad s_{2}=(2 k+1) \max \left(m_{2}^{+}, m_{2}^{-}\right),
\end{aligned}
$$

where the last follows from the estimate

$$
\mu \int_{\mu^{m} / \varepsilon^{2 k+1}}^{\infty} \exp \left(-\frac{t^{2 k+1}}{2 k+1}\right) d t \leqq K^{*} \varepsilon^{\frac{1}{m(2 k+1)}}
$$

Then, because of $s_{2} \geqq 2 k+1$; the barrier function

$$
K_{1} \varepsilon^{\frac{1}{s_{1}}}-K_{2} \varepsilon^{\frac{1}{2 k+1}} v_{\ell}\left(x_{2}\right)
$$

yields Theorem 2.2: Let $L_{0} \equiv-x_{2}{ }^{2 k} \frac{\partial}{\partial x_{2}}$ and let the singular line belong to the boundary
of $\Omega$. Then it holds that

$$
\left\|u_{e}-\left(g_{2}+v\right)\right\| \leqq K \varepsilon^{\frac{1}{B_{i}}}
$$

with $s_{2}=(2 k+1) \max \left(m_{2}^{+}, m_{2}^{-}\right)$.

The case

$$
\Omega=\left\{\left(x_{1}, x_{2}\right) \mid-1<x_{1}<+1,-f_{1}\left(x_{1}\right)<x_{2}<0\right\}
$$

is analogous to that which has been considered first. Setting $\left.g\right|_{x_{2}=0}=g_{0}\left(x_{1}\right)$ we obtain $\left\|u_{\varepsilon}-\left(g_{0}+v\right)\right\| \leqq K \varepsilon^{\frac{1}{s_{2}}}$ with $s_{3}=(2 k+1) \max \left(m_{1}^{+}, m_{1}^{-}\right)$.

Now let $l$ be odd, $l=2 k-1$. In this case the sign of $\pm x_{2}{ }^{2 k-1} \frac{\partial}{\partial x_{2}^{\prime}}$ is of decisive importance. First we consider

with

$$
\begin{aligned}
L_{\varepsilon} u_{\varepsilon} \equiv \varepsilon L_{1} u_{\varepsilon}-x_{2}{ }^{2 k-1} \frac{\partial u_{\varepsilon}}{\partial x_{2}} & =0 \text { in } \Omega \\
u_{\varepsilon} & =g \text { on } \partial \Omega
\end{aligned}
$$

$$
\Omega=\left\{\left(x_{1}, x_{2}\right) \mid-1<x_{1}<+1,-f_{1}\left(x_{1}\right)<x_{2}<f_{2}\left(x_{1}\right)\right\} .
$$


Because of $\Gamma_{-}=\partial \Omega$ the solution of the global problem becomes .

$$
u=\left\{\begin{array}{ll}
g_{2}\left(x_{1}\right), & x_{2}>0 \\
g_{1}\left(x_{1}\right), & x_{2}<0
\end{array} .\right.
$$

and at $x_{2}=0$ a free boundary layer originates. By setting $\zeta=\frac{x_{2}}{\varepsilon^{\frac{1}{2 k}}}$ in the neigh-
bourhood of $x_{2}=0$ we obtain

$$
L_{\varepsilon}=\varepsilon^{\frac{2 k-1}{2 k}}\left\{-a\left(x_{1}\right) \frac{\partial^{2}}{\partial \zeta^{2}}-\zeta^{2 k-1} \frac{\partial}{\partial \zeta}\right\}+\varepsilon^{\frac{2 k-1}{2 k}} L_{\varepsilon}^{*} .
$$

The requirements

$$
a \frac{\partial^{2} v}{\partial \zeta^{2}}+\zeta^{2 k-1} \frac{\partial v}{\partial \zeta}=0,\left.\quad v\right|_{\zeta \doteq-\infty}=\frac{g_{1}-g_{2}}{2},\left.\quad v\right|_{\zeta=+\infty}=\frac{g_{2}-g_{1}}{2}
$$

yield

$$
v=\frac{g_{2}-g_{1}}{2 A^{*}} \int_{0}^{\infty} \exp \left(-\frac{1}{a 2 k} t^{2 k}\right) d t \text { with } A^{*}=\int_{0}^{\infty} \exp \left(-\frac{1}{a 2 k} t^{2 k}\right) d t
$$

Theorem 2.3: Let $L_{0} \equiv-x_{2}{ }^{2 k-1} \frac{\partial}{\partial x_{2}}$ and let the singular line lie in the interior
$\Omega$. Then it holds that

$$
\left\|u_{\varepsilon}-\left(\frac{g_{2}+g_{1}}{2}+v\right)\right\| \leqq K \begin{cases}\varepsilon^{\frac{1}{2}}|\ln \varepsilon| & \text { if } k=1 \text { and } s_{4}=2 \\ \frac{1}{\varepsilon^{s_{4}}} & \text { otherwise }\end{cases}
$$

with $s_{4}=2 k \max \left(m_{2}^{+}, m_{2}^{\prime}, m_{1}^{+}, m_{1}^{-}\right)$.

Proof: According to the construction we have

$$
\left|L_{c}\left(u_{\varepsilon}-\left(\frac{g_{2}+g_{1}}{2}+v\right)\right)\right| \leqq K_{\varepsilon}^{\frac{2 k-1}{2 k}} \text { in } \Omega .
$$

Since $v$ is a function of boundary layer type, it follows that

$$
\frac{g_{2}+g_{1}}{2}+\left.v\right|_{r_{-}}=g+r \text { with }|r| \leqq K \varepsilon^{N} \quad(N \text { arbitrary })
$$

in the domain $|x| \leqq x_{0}<1$. However, analogous to the above, we obtain only

$$
\left\|u_{\varepsilon}-\left(\frac{g_{2}+g_{1}}{2}+v\right)\right\|_{r_{-}} \leqq \bar{K}^{\frac{1}{s_{c}}}
$$

with $s_{4}=2 k \max \left(m_{2}{ }^{+}, m_{2}^{-}, m_{1}{ }^{+}, m_{1}^{-}\right)$. Now, we can conclude that $K_{1} \varepsilon^{\frac{1}{s_{4}}} \perp K_{2} \varepsilon^{\frac{1}{2 k}}$. $\times w_{c}\left(x_{2}\right)$ and $K_{1} \varepsilon^{\frac{1}{2}}|\ln \varepsilon|-K_{2} \varepsilon^{\frac{1}{2}} w_{c}\left(x_{2}\right)$ if $k=1$ and $s_{4}=2$, respectively, with

$$
w_{e}\left(x_{2}\right)=\int_{0}^{x_{2} / e^{\frac{1}{2 k}}} \int_{0}^{q} \exp \left(\frac{1}{K_{3}}\left(p^{2 k}-q^{2 k}\right)\right) d p d q
$$


are barrier functions for $u_{\varepsilon}-\left(\frac{g_{2}+g_{1}}{2}+v\right)$. Because of

$$
\left|w_{\varepsilon}\left(x_{2}\right)\right| \leqq \dot{K}|\ln \varepsilon| \text { for } \dot{k}=1 \text { and }\left|w_{\varepsilon}\left(x_{2}\right)\right| \leqq K \text { for } \dot{k}>1
$$

we obtain the assertion of Theorem 2.3

In the case $\Omega=\left\{\left(x_{1}, x_{2}\right) \mid-1<x_{1}<+1,0<x_{2}<f_{2}\left(x_{1}\right)\right\}$ a boundary layer of the above type appears and it does not change so much.

Now we consider an other type of a singularly perturbed problem, namely

$$
\begin{aligned}
L_{\varepsilon} u_{\varepsilon} \equiv \varepsilon L_{1} u_{\varepsilon}+x_{2}^{2 k-1} \frac{\partial u_{\varepsilon}}{\partial x_{2}} & =0 \text { in } \Omega \\
u_{\varepsilon} & =g \text { on } \partial \Omega
\end{aligned}
$$

where $\Omega=\left\{\left(\ddot{x}_{1}, x_{2}\right) \mid-1<x_{1}<+1,-f_{1}\left(x_{1}\right) \leqslant x_{2}, \ldots f_{2}\left(x_{1}\right)\right\}$. Here we have.

$$
\Gamma_{+}=\partial \Omega \backslash\{(1,0) \cup(-1,0)\} \text {. }
$$

Therefore we cannot impose conditions on the solution of the global problem $U\left(x_{1}\right)$. Let $v^{1,2}$ be the local correctors in the neighbourhood of the boundary, such that $\left|L_{\varepsilon} v^{1,2}\right| \leqq K_{\varepsilon}{ }^{2}$ and $v^{1,2}+U$ satisfy the boundary conditions for $x_{2} \stackrel{x_{2}}{=}\left(x_{1}\right)$ and $x_{2}=-f_{1}\left(x_{1}\right)$, respectively. Then, it holds.

$$
v^{1,2}=v_{0}^{1,2}+\varepsilon v_{1}^{1,2}+\varepsilon^{2} v_{2}^{1,2}
$$

with

$$
\begin{aligned}
& v_{0}^{2}=\left(g_{1}-U\right) \exp \left(-\frac{f_{1}^{2 k-1}}{a} \frac{f_{1}\left(x_{1}\right)+x_{2}}{\varepsilon}\right) \\
& v_{0}^{2}=\left(g_{2}-U\right) \exp \left(-\frac{f_{2}^{2 k-1}}{a} \frac{f_{2}\left(x_{1}\right)-x_{2}}{\varepsilon}\right)
\end{aligned}
$$

If $a_{11}, a_{1}$ and $a_{0}$ do not depend on $x_{2}$, then we can improve the accuracy of the approximation by requiring

$$
a_{11} \frac{\partial^{2} U}{\partial x_{1}^{2}}+a_{1} \frac{\partial U}{\partial x_{1}}+a_{0} U=0 .
$$

If follows $\left|L_{\varepsilon}\left(u_{\varepsilon}-\left(\dot{U}+v^{1}+v^{2}\right)\right)\right| \leqq K \varepsilon^{2}$.

In the domain $|x| \leqq x_{0}<1 v_{0}^{1}$ and $v_{0}^{2}$ are again exponentially small for $x_{2}==f_{2}\left(x_{1}\right)$ and $x_{2}=-f_{1}\left(x_{1}\right)$, respectively. In the neighbourhood of $\left|x_{1}\right|=1$ we obtain the conditions

$$
U(1)=g(1) \text { and } \quad U(-1)=g(-1)
$$

\section{by requiring}

$$
\left|\hat{v}_{0}{ }_{x_{2}=f_{2}\left(x_{1}\right)}\right|+\left|v_{0}^{2}\right|_{x_{1}=-f_{1}\left(x_{1}\right)} \mid \leqq K \varepsilon^{\frac{2}{g}} \quad(s>0) \text {. }
$$

Since $a_{0} \geqq 0$ we can determine the solution $U\left(x_{1}\right)$ of the global problem as the unique solution of the boundary value problem

$$
\begin{aligned}
& a_{11} \frac{\partial^{2} U}{\partial x_{1}^{2}}+a_{1} \frac{\partial U}{\partial x_{1}}+a_{0} U=0 \\
& U(1)=g(1), \quad U(-1)=g(-1) .
\end{aligned}
$$


For $s$ we obtain, as above, that

$$
\begin{array}{r}
s=s_{5}=\max \left(\min \left(m_{1}{ }^{+}, m_{2}{ }^{+}\right)+l m_{1}{ }^{+}, \min \left(m_{1}{ }^{+}, m_{2}{ }^{+}\right)+l m_{2}{ }^{+},\right. \\
\left.\min \left(m_{1}{ }^{-}, m_{2}{ }^{-}\right)+l m_{1}{ }^{-}, \min \left(m_{1}{ }^{-}, m_{2}^{-}\right)+l m_{2}^{-}\right) .
\end{array}
$$

By means of the barrier function

$$
K_{1} \varepsilon^{\frac{1}{s *}}-K_{2} \varepsilon \exp \left(-K_{3} x_{1}\right)
$$

we immediately obtain

Theorem 2.4: Let $L_{0}=x_{2}{ }^{2 k-1} \frac{\partial}{\partial x_{2}}$ and let the singular line lie in the interior of the domain $\Omega$. Furthermore, let $a_{11} ; a_{1}, a_{0}$ be independent of $x_{2}$. Then it holds

$$
\left\|u_{\varepsilon}-\left(U+v_{0}^{1}+v_{0}^{2}\right)\right\| \leqq K \varepsilon^{\frac{1}{s_{0}}}
$$

with the above defined $s_{5}$, where $U\left(x_{1}\right)$ denotes the solution of the boundany value problem (2.2).

Remark: In the case $k=1 a_{11}, a_{1}$ and $a_{0}$ can also depend on $x_{2}$. Then, we require

$$
a_{11}\left(x_{1}, 0\right) \frac{\partial^{2} U}{\partial x_{1}^{2}}+a_{1}\left(x_{1}, 0\right) \frac{\partial U}{\partial x_{1}}+a_{0}\left(x_{1}, 0\right) U=0
$$

set $\Phi_{\varepsilon}=U\left(x_{1}\right)+\varepsilon U_{1}\left(x_{1}, x_{2}\right)+v^{1}+v^{2}$ and determine $U_{1}$ from

$$
\begin{aligned}
x_{2} \frac{\partial U_{1}}{\partial x_{2}}= & {\left[a_{11}\left(x_{1}, x_{2}\right)-a_{11}\left(x_{1}, 0\right)\right] \frac{\partial^{2} U}{\partial x_{1}^{2}}+\left[a_{1}\left(x, x_{2}\right)-a_{1}\left(x_{1}, 0\right)\right] \frac{\partial U}{\partial x_{1}} } \\
& +\left[a_{0}\left(x_{1}, x_{2}\right)-a_{0}\left(x_{1}, 0\right)\right] C .
\end{aligned}
$$

It always hold $s \geqq 2$. $s=2$ holds if and only if $m_{1}^{+}:=m_{2}{ }^{+}=m_{1}^{-}=m_{2}^{-}=1$ and $k=1$. The assertion of Grasman [6], that $s=1$ for $k=1$, is not valid.

The cases

$$
\Omega=\left\{\left(x_{1}, x_{2}\right) \mid 0<x_{2}<f_{2}\left(x_{1}\right)\right\} \text { and } \Omega=\left\{\left(x_{1}, x_{2}\right) \mid-f_{1}\left(x_{1}\right)<x_{2}<0\right\},
$$

respectively, are less interesting since the solutions of the global problem, namely $g_{1}\left(x_{1}\right)$ and $g_{2}\left(x_{1}\right)$, respectively are uniquely determined.

\section{A singular line parallel to the characteristies}

We now consider

$$
\begin{aligned}
L_{\varepsilon} u_{\varepsilon}=\varepsilon L_{1} u_{\varepsilon}+x_{2}^{l} \cdot \frac{\partial u_{\varepsilon}}{\partial x_{1}} & =0 \text { in } \Omega \\
u_{\varepsilon} & =g \text { on } \partial \Omega
\end{aligned}
$$

with $\Omega=\left\{\left(x_{1}, x_{2}\right) \mid-1<x_{1}<+1,0<x_{2}<f\left(x_{1}\right)\right\}$ and assume that the lines $x_{2}=$ const. intersect $\partial \Omega$ at exactly two points (except $x_{2}=0$ and $x_{2}=\sup f\left(x_{1}\right)$ ). Furthermore, let us assume that $f\left(x_{1}\right)<f(0)$ for all $x_{1} \neq 0$, such that

$$
\begin{aligned}
& \Gamma_{-}=\left\{\left(x_{1}, x_{2}\right) \mid-1<x_{1}<0, x_{2}=f\left(x_{1}\right)\right\} \\
& \Gamma_{+}=\left\{\left(x_{1}, x_{2}\right) \mid 0<x_{1}<+1, x_{2}=f\left(x_{1}\right)\right\}
\end{aligned}
$$


Let $u$ be the solution of the global problem

$$
\frac{\partial u}{\partial x_{1}}=0,\left.\quad u\right|_{r_{-}}=g
$$

$u$ is a smooth function in $\bar{\Omega}$ except a neighbourhood of $(0, f(0))$. Near the singular line $x_{2}=0$ let $\zeta=\frac{x_{2}}{\frac{1}{\varepsilon^{2+l}}}$ and let $v$ satisfy

$$
a_{22}\left(x_{1}, 0\right) \frac{\partial^{2} v}{\partial \zeta^{2}}-\zeta^{l} \frac{\partial v}{\partial x_{1}}=0,\left.\quad v\right|_{\zeta=0}=g-\left.u\right|_{x_{2}=0}
$$

This boundary layer problem has been studied in [16], because of the singularity of $\frac{\partial^{2} v}{\partial x_{1} \partial \zeta}, \frac{\partial^{2} v}{\partial x_{1}^{2}}$ at $(-1,0)$ a regularization method has been applied.

Theorem 3.1: Let $\Omega_{\gamma}=\Omega \cap\left\{x \mid\right.$ dist $\left.\left(x, I_{+}\right)>\dot{\gamma}\right\}$. Then it holds

$$
\left\|u_{\varepsilon}-(u+v)\right\|_{\bar{o}_{y}} \leqq K \varepsilon^{\frac{1}{2(2+l)}}
$$

Proof: In $\Omega_{\frac{x}{2}}$, for corresponding regularization, we have

$$
\begin{aligned}
& \left|L_{\varepsilon}\left(u_{\varepsilon}-(u+v)\right)\right| \leqq K \varepsilon^{1-\frac{3}{2(2+) 1}} \\
& \left|u_{\varepsilon}-(u+v)\right|_{x_{2}=0} \leqq K \varepsilon^{\frac{1}{2(2+1)}}, \quad\left|u_{\varepsilon}-(u+v)\right|_{r_{-}}=0, \quad\left|u_{\varepsilon}-(u+v)\right| \leqq K
\end{aligned}
$$

according to the construction. Now, let $\psi=\psi\left(x_{1}, x_{2}\right)$ be a smooth function with $\psi \equiv 0$ in $\Omega_{\gamma}, \psi \equiv 1$ in,$\Omega \backslash \Omega_{\frac{\gamma}{2}}$ and $\frac{\partial \psi}{\partial x_{1}} \geqq 0$. We construct a barrier fupction $S_{\varepsilon}$ by
setting

with

$$
S_{\varepsilon}\left(x_{1}, x_{2}\right)=K_{1} \psi\left(x_{1}, x_{2}\right)+K_{2} \frac{1}{2(2+l)} \exp \left(K_{3} x_{1}\right)\left(K_{4}+: V_{\varepsilon}\left(x_{2}\right)\right)
$$

$$
V_{\varepsilon}\left(x_{2}\right)=\varepsilon^{-\frac{1}{2(2+l)}} x_{2}^{\frac{1}{2}} S_{\frac{2-l}{2(2+l)} \cdot \frac{1}{2+l}}\left(\frac{2}{2+l} x_{2}^{\frac{2+l}{2}} \varepsilon^{-\frac{1}{2}}\right)
$$

and appropriately chosen constants $K_{1}, K_{2} ; K_{3}, K_{4} . S_{p, q}(\cdot)$ denotes the Lommel function. As a result we obtain that $S_{\varepsilon}$ majorizes $u_{\varepsilon}-(u+v)$ in $\Omega_{\frac{y}{2}}$. The restriction
to $\Omega_{y}$ yields the assertion

Remark: Of course, it is possible to construct a local corrector in the neighbourhood of $\Gamma_{+}$. For the study of the asymptotic behaviour in the neighbourhood of the points $(0, f(0))$ and $(1,0)$ we refer to $[14,9]$.

The case $\Omega=\left\{\left(x_{1}, x_{2}\right) \mid-1<x_{1}<+1,-f_{1}\left(x_{1}\right)<x_{2}<f_{2}\left(x_{1}\right)\right\}$ and $l$ even is not interesting, since nothing happens along the singular line. However, if $l=$ odd, the problem becomes more difficult. For $l=1$ this case have been considered by BartoN [1] and Gorkov [5]. 


\section{REFERENCES}

[1] Bartón, N. G.: On the asymptotic solution of an elliptic interior layer problem. J. Austral. math. Soc. Ser. B 19 (1976), 493-512.

[2] De Groes, P. P. N.: Singularly perturbed differential operators of second order. Math. Centre Tracts 68. Amsterdam 1976.

[3] DE JAGER, E. M.: Singular elliptic perturbation of vanishing first-order differential operators. Lect. Notes Math. 280 (1972), 73-86.

[4] Devinatz, A., and A. Friedman: The asymptotic behaviour of the solution of a singularly perturbed Dirichlet problem. Indiana Univ. Math. J. 27 (1978), 527-537.

[5] Горьков, Ю. П.: Об одном применении метода сращивания асимптотических разложений. ДАН СССР $\mathbf{2 5 3}$ (1980), 786-790.

[6] Grasman, J.:'Elliptic singular perturbations of first order differential operatòrs vanishing at an interior surface. MC Amsterdam.TW 169/77 (1977).

[7] Grasman, J.: On a class of clliptic singular perturbations with applications in population genetics. Math. Methods Appl. Sci. 1 (1979), $432-441$.

[8] Grasman, J., and B. J. Matrowski: A variational approach to singularly perturbed boundary value problems for ordinary and partial differential equations with turning points. SIAM J. Appl. Math. 32 (1977), 588-597.

[9] Ильин, А. М., и Е. Ф. ЛЕликовА: Метод сращивания асимптотических разложений для уравнения $\varepsilon \Delta u-a(x, y) u_{\nu}=f(x, y)$ в прлмоугольнике. Мат. сб. 96 (138), $4(1975), 568-583$.

[10] КАлякин, Л. А.: Асимптотика решения әллиптической задачи с сингулярным возмущением в случае седловоп̆ точки у предельного оператора. Тр. Ин-та Мат. и Мех. УНЦ АН СССР $\mathbf{2 8}(1979), 15-39$.

[11] KAMN, S.: Elliptic perturbation of a first order operator with a singular point of attracting type. Indiana Univ. Math. J. 27 (1978), 935-952.

[12] Kamis, S.: On elliptic singular perturbation problems with turning points. SIAM J. Math. Anal. 10 (1979), 447-455.

[13] Kamin, S.: On elliptic equations with a small parameter in the highest derivatives. Comm. in part. diff. equ. 4 (6) (1979), 573-593.

[14] ЛЕликовм, Е. Ф.: Об асимптотике решения эллиптического уравнения второго порядка с малым параметром при старших производных. Дифф. уравнения 12 $(1976), 1852-1865$.

[15] Miciael, J. H.: A general theory for linear elliptic partial differential equations. J. diff. equ. 23 (1977), 1 - 29.

[16] TовіsкA, L.: Die asymptotische Lösung einer linearen elliptischen Differentialgleichung mit Wendepunkten. Beiträge zur Analysis 13 (1979), 77-82.

[17] TовısкA, L.: A priori Abschätzungen für singulär gestörte elliptische Probleme zweiter . Ordnung. Beiträge zur Analysis 17 (1981), 41-48.

Manuskripteingang: 18.06. 1982

\section{VERFASSTER:}

Dr. H.-G. Roos und Dr. L. Toвiska

Sektion Mathematik und Physik der TH „Otto von Guericke“

DDR - 3040 Magdeburg, Boleslaw-Bierut-Platz 